\title{
Experimental Constraints in the Study of the Biosynthesis of Indole Alkaloids in Fungi
}

\author{
By IAN LAWS AND PETER G. MANTLE* \\ Department of Biochemistry, Imperial College of Science, Technology and Medicine, \\ London SW7 $2 A Y, U K$
}

(Received 16 December 1988; revised 6 April 1989; accepted 31 May 1989)

\begin{abstract}
The disproportionate difficulty in obtaining compelling experimental evidence from ${ }^{14} \mathrm{C}$-radiolabelling that the indole moiety of the otherwise isoprenoid penitrem $\mathrm{A}$ is biosynthesized by Penicillium crustosum directly from tryptophan has been explored. [benzene ring- ${ }^{14} \mathrm{C}$ ] Tryptophan added to the broth beneath the mycelial mat of stationary liquid cultures labelled penitrem $\mathrm{A}$ with $1.4 \%$ incorporation, only threefold more than that determined for [methylene$\left.{ }^{14} \mathrm{C}\right]$ tryptophan or $\left[\mathrm{U}-{ }^{14} \mathrm{C}\right]$ tyrosine, incorporation of which could only have been indirect. In contrast, the substituted tryptophan-histidine diketopiperazine roquefortine, biosynthesized concurrently with penitrems by this organism, was labelled with compelling efficiency $(23.4 \%$ incorporation of [benzene ring $\left.{ }^{14} \mathrm{C}\right]$ tryptophan). In submerged culture, Claviceps paspali concurrently biosynthesized an analogous pair of metabolites, 3-hydroxy-3-methylbutenyl paspalinine and lysergic acid $\alpha$-hydroxyethylamide. This feature enabled experimental demonstratation of [benzene ring- ${ }^{14} \mathrm{C}$ ]tryptophan incorporation to an extent more consistent with direct contribution of the indole moiety of the indole-diterpenoid paspalinine derivative. The same precursor applied to the sporing surface of $P$. crustosum stationary cultures also provided stronger evidence for a direct biosynthetic role in the formation of penitrem $A$. In the absence of competition from any other indolic secondary metabolite, a submerged culture of Penicillium paxilli incorporated $5 \%$ of the [benzene ring $\left.-{ }^{14} \mathrm{C}\right]$ tryptophan given during growth into the indole-diterpenoid paxilline. A double-labelling time-course experiment indicated temporal separation of steps in the biosynthesis of roquefortine. The inadequacy of classical precursor techniques for studying biosynthesis of indole-diterpenoids in P. crustosum is discussed. The more homogeneous submerged culture fermentation system is preferred for experimentation.
\end{abstract}

\section{INTRODUCTION}

A biologically significant group of fungal secondary metabolites, comprising an indole nucleus substituted with isoprenoid moieties, has become apparent with the sequential elucidation of the structures of paxilline (Springer et al., 1975), the paspalinines (Cole et al., 1977), aflatrem (Gallagher et al., 1980 b), the penitrems (de Jesus et al., 1981) and the janthitrems (de Jesus et al., 1984). Lolitrem B, occurring so far only as a natural product of fungusendophyte-infected ryegrass (Gallagher $e$ et al., 1984), is structurally analogous to this group and is probably elaborated at least in part by the ryegrass endophyte Acremonium loliae (Weedon \& Mantle, 1987). Many of these compounds are potently tremorgenic in animals so that, for example, penitrem A was implicated (Mantle et al., 1978) as a possible aetiological agent in ryegrass staggers, a neurological disorder of sheep and cattle in New Zealand. The suggestion, based partly on the evidence of experimental pathology, stimulated attempts at Imperial College to elucidate the penitrem A structure by spectroscopic and biosynthetic techniques. However, convincing experimental evidence to support a contention that the indole nucleus of the penitrem molecule was derived in Penicillium crustosum directly from tryptophan seemed impossible to obtain using ${ }^{14} \mathrm{C}$-labelled putative precursor. This was the more inexplicable since 
the isoprenoid moieties were so readily biosynthetically labelled experimentally from $\left[{ }^{14} \mathrm{C}\right]$ or $\left[{ }^{13} \mathrm{C}\right]$ mevalonate. Thus, when the structure of penitrem A was first proposed (de Jesus et al., 1981 ) on the basis of spectroscopic and biosynthetic reasoning, the simple statement that the incorporation of radiolabel from [benzene ring ${ }^{-14} \mathrm{C}$ ] tryptophan $(0 \cdot 16 \%)$ into penitrem $\mathrm{A}$ was eightfold greater than that of [methylene $\left.{ }^{-14} \mathrm{C}\right]$ tryptophan (which is impossible to incorporate directly) was hardly compelling evidence for the direct derivation of the penitrem indole from tryptophan. This was especially so as no more detailed experimental evidence was given, nor has since been published. The question remains, is eight times a spurious value, due only to metabolic scrambling of the radiolabel in the putative precursor, compelling evidence? Thus, while the structure proposed by de Jesus et al. (1981) was never in dispute, the biosynthetic origin of the indole could not be regarded as experimentally proven.

The present studies were initiated in 1982 to address this problem using surface liquid culture of $P$. crustosum, the only system available at that time for the biosynthesis of the penitrem tremorgens. Subsequently, de Jesus et al. (1983) presented brief evidence from ${ }^{13} \mathrm{C}$-labelling studies, arising out of other studies on the biosynthesis of roquefortine by $P$. crustosum (Steyn \& Vleggaar, 1983), which confirmed that the penitrem indole was derived directly from tryptophan. Again, no experimental details such as the extent of ${ }^{13} \mathrm{C}$-signal enrichment were published, but the present authors accepted the evidence as definitive. The present studies, therefore, were initially conditioned by the possibility that a novel alternative biosynthetic pathway was operating as a source of the penitrem indole. Later, using a classical experimental approach, our efforts were directed solely at explaining the difficulty of obtaining compelling biosynthetic evidence of conventional tryptophan secondary metabolism in a fungus.

The closely concurrent production of roquefortine, a substituted tryptophan-histidine diketopiperazine in which the whole carbon skeleton of tryptophan is retained (Barrow et al., 1979), in the penitrem-producing $P$. crustosum appeared to provide a strategic internal standard against which incorporation into penitrem $A$ of tryptophan radiolabelled in the indole moiety could be measured. It has enabled the present dilemma of the inconsistent applicability of classical biosynthetic methods in fungi to be given a quantitative dimension, and emphasizes the neglected study of comparative biosynthesis dynamics in microbial secondary metabolism, particularly with respect to metabolites of eukaryotic micro-organisms, in which the widest range of structural and mechanistic diversity is expressed.

\section{METHODS}

Organisms. The strain of Penicillium crustosum Thom was isolated from New Zealand soil and has been used in previous studies (Mantle et al., 1983). Penicillium paxilli Bain. was as previously described (Ibba $e$ t al., 1987) and the strain of Claviceps paspali Stevens et Hall used was ATCC 13892. Cultures were maintained on potato dextrose agar.

Media. The liquid media for Penicillium spp. were based on Czapek Dox broth supplemented with yeast extract (CDYE broth), pH 6.8, containing ( $\mathrm{g} \mathrm{l}^{-1}$ in distilled water); sucrose, $30 ; \mathrm{NaNO}_{3}, 3 ; \mathrm{KH}_{2} \mathrm{PO}_{4}, 1 ; \mathrm{MgSO}_{4} .7 \mathrm{H}_{2} \mathrm{O}$, $0.5 ; \mathrm{KCl}, 0.5 ; \mathrm{FeSO}_{4} .7 \mathrm{H}_{2} \mathrm{O}, 0.01$; yeast extract (Difco), 5 . A variation increased yeast extract to $20 \mathrm{~g} \mathrm{l}^{-1}$.

For $C$. paspali the primary stage medium contained $\left(\mathrm{g} \mathrm{l}^{-1}\right.$ in tap water): mannitol, $40 ; \mathrm{KH}_{2} \mathrm{PO}_{4}, 1$; $\mathrm{MgSO}_{4} .7 \mathrm{H}_{2} \mathrm{O}, 0.3$; chick pea meal, 1 ; succinic acid, $10 ; \mathrm{pH}$ adjusted to $5 \cdot 2$ with ammonium hydroxide solution (0.88 sp.gr.). The production stage medium contained ( $\mathrm{g} \mathrm{l}^{-1}$ in tap water): mannitol, $50 ; \mathrm{KH}_{2} \mathrm{PO}_{4}, 1$; $\mathrm{MgSO}_{4} .7 \mathrm{H}_{2} \mathrm{O}, 0 \cdot 3$; succinic acid, $30 ; \mathrm{pH}$ adjusted to 5.2 with ammonium hydroxide solution ( 0.88 sp.gr.).

Liquid culture fermentations. All fermentations used $100 \mathrm{ml}$ medium in $500 \mathrm{ml}$ Erlenmeyer flasks. P. crustosum spores were scattered over the surface of the medium and the cultures incubated at $27^{\circ} \mathrm{C}$. $P$. paxilli sporing mycelium from a $15 \mathrm{ml}$ potato dextrose agar (PDA) slope culture $(10 \mathrm{~d})$ was macerated in CDYE broth in a baffled Erlenmeyer flask and incubated at $27^{\circ} \mathrm{C}$ for $24 \mathrm{~h}$ on a rotary shaker $(200$ r.p.m., $10 \mathrm{~cm}$ eccentric throw). Then $10 \mathrm{ml}$ volumes of this primary-stage culture were transferred to production-stage (unbaffled) flasks and incubated on a rotary shaker at $27^{\circ} \mathrm{C}$. C. paspali mycelium from an $8 \mathrm{~d}$ PDA culture was macerated in primary-stage medium and incubated at $24^{\circ} \mathrm{C}$ for $7 \mathrm{~d}$ on a rotary shaker. The culture was homogenized aseptically (Sorvall Omnimixer) and $10 \mathrm{ml}$ volumes were transferred to production-stage flasks and incubated on a rotary shaker at $24^{\circ} \mathrm{C}$.

Reference samples of metabolites. Authentic samples of penitrems, roquefortine, cyclopenin, paxilline, paspalinine and 3-hydroxy-3-methylbutenyl paspalinine were prepared by the methods described and their identity confirmed by electron impact mass spectrometry and ${ }^{1}$ H NMR spectroscopy. Lysergic acid $\alpha$-hydroxyethylamide was supplied by Farmitalia, Milan.

Radiochemicals. Radiochemicals (Amersham) were DL-[benzene ring-U- $\left.{ }^{14} \mathrm{C}\right]$ tryptophan $\left(84 \mathrm{mCi} \mathrm{mmol}^{-1}\right)$, 
DL- $\left[\right.$ methylene $\left.{ }^{-14} \mathrm{C}\right]$ tryptophan $\left(53.5 \mathrm{mCi} \mathrm{mmol}^{-1}\right), \quad \mathrm{DL}-\left[2-{ }^{-14} \mathrm{C}\right] \mathrm{mevalonic}$ acid lactone $\left(53 \mathrm{mCi} \mathrm{mmol}^{-1}\right)$, L-[U-14 C]phenylalanine $\left(496 \mathrm{mCi} \mathrm{mmol}^{-1}\right), \mathrm{L}-\left[\mathrm{U}^{-14} \mathrm{C}\right]$ tyrosine $\left(504 \mathrm{mCi} \mathrm{mmol}^{-1}\right),\left[\right.$ carboxyl- $\left.{ }^{14} \mathrm{C}\right]$ anthranilic acid (10.5 mCi mmol $\left.{ }^{-1}\right), \quad \mathrm{D}-\left[2,3,4,5-(\mathrm{n})^{-14} \mathrm{C}\right] \mathrm{shikimic}$ acid $\left(250 \mathrm{mCi} \mathrm{mmol}^{-1}\right), \quad \mathrm{DL}-\left[2-{ }^{-3} \mathrm{H}_{3}\right] \mathrm{mevalonic}$ acid lactone $\left(1.3 \times 10^{3} \mathrm{mCi} \mathrm{mmol}^{-1}\right)$, L-[ring-2-14 $\mathrm{C}^{-}$histidine $\left(59 \mathrm{mCi} \mathrm{mmol}^{-1}\right)\left(1 \mathrm{Ci} \equiv 3.7 \times 10^{10} \mathrm{~Bq}\right)$. Radiochemicals in sterile aqueous solution were injected into the broth of stationary liquid cultures by piercing the mycelial mat with

a Pasteur pipette. Solutions were added directly to submerged cultures.

Radioactivity measurement. The radioactivity in purified metabolites was measured by liquid scintillation counting (Kontron) of samples, in $100 \mu \mathrm{l}$ methanol, using toluene/naphthalene/2(4-t-butylphenyl)-5-(4biphenylyl)-1,3,4-oxadiazole scintillant $(4 \mathrm{ml})$. Counting efficiencies were determined using radiolabelled hexadecane.

Autoradiography. Chromatography plates were overlaid with X-ray film (Fuji, RX Medical), secured between glass plates and stored at $-70^{\circ} \mathrm{C}$ for the desired period before developing (Kodak X-0-MAT).

Silica layer chromatography. Thin-layer $(0.25 \mathrm{~mm})$ chromatography (TLC) used Polygram SIL/UV 254 plates (Camlab). Preparative layer plates were prepared on glass using silica gel $60 \mathrm{GF}_{254}$ (Merck) at a depth of $1 \mathrm{~mm}$. Extracts were loaded in chloroform and chromatograms developed using chloroform/acetone (93:7, v/v). Location of metabolites of interest at $254 \mathrm{~nm}$ was confirmed where necessary by spraying with $3 \%(\mathrm{w} / \mathrm{v}) \mathrm{FeCl}_{3}$ in n-butanol. Penitrems A and E coloured green; cyclopenin, blue; ergosterol, green becoming blue/grey on gentle heating; paxilline, green. Paspalinine and 3-hydroxy-3-methylbutenyl paspalinine were revealed by the blue colour in response to spraying with $50 \%(\mathrm{v} / \mathrm{v})$ ethanolic $\mathrm{H}_{2} \mathrm{SO}_{4}$. The silica in regions of interest was transferred from the plate to a glass column $(1 \times 15 \mathrm{~cm})$ and eluted with methanol which was concentrated to small volume in vacuo prior to HPLC.

Preparative HPLC. Rapid preparation of multi-milligram amounts of penitrems $\mathrm{A}$ and $\mathrm{E}$ was achieved using a Waters $\mu$-bondapak $\mathrm{C}_{18}(10 \mu \mathrm{m}$ particle size $)$ cartridge $(100 \times 8 \mathrm{~mm})$ in a Z-module, methanol/water $(5: 1, \mathrm{v} / \mathrm{v})$ mobile phase at $4-5 \mathrm{ml} \mathrm{min}-1$ and UV detection $(233 \mathrm{~nm})$. Injection volumes $(20 \mu \mathrm{l}$ in methanol) were resolved so that penitrems $A$ and E eluted 3-5 min after injection. Further isolation of penitrem A used an Altex Ultrasphere ODS $(5 \mu \mathrm{m}$ particle size $)$ column $(250 \times 10 \mathrm{~mm})$ which with similar mobile phase gave baseline separation of penitrems $A$ and $E$, with mean retention times of 7 and $5 \mathrm{~min}$, respectively.

Preparation of roquefortine used the Ultrasphere ODS column, methanol/water $(4: 1, \mathrm{v} / \mathrm{v})$, flow rate 3-4 ml min ${ }^{-1}$, detector wavelength $325 \mathrm{~nm}$ and $20-50 \mu \mathrm{l}$ injection volumes in methanol. Roquefortine eluted 8-10 min after injection.

Crude cyclopenin, isolated by preparative layer chromatography, was purified as for roquefortine except using a flow rate of $2.5-3.0 \mathrm{ml} \mathrm{min}^{-1}$ and a detector wavelength of $290 \mathrm{~nm}$. Cyclopenin eluted 7-8 min after injection.

Analytical procedures. The metabolites of interest from Penicillium spp. were located principally in the cells which were separated by filtration from submerged cultures and by decanting the underlying broth of stationary cultures. The undersides of fungal mats were washed with distilled water, and the biomass was homogenized in distilled water (Sorvall Omnimixer) and freeze-dried to constant weight prior to extraction.

$C$. paspali metabolites were located in the broth (lysergic acid derivatives) and in the mycelium (indolediterpenoids) which were separated by filtration. The mycelium was freeze-dried to constant weight before extraction; the broth was extracted directly by liquid-liquid partition.

(i) Mycelial dry weight. The mycelium from each culture flask was freeze-dried to constant weight.

(ii) Extraction of Penicillium spp. metabolites. Freeze-dried biomass from one Erlenmeyer flask culture $(\sim 1 \mathrm{~g})$ was extracted with acetone $(200 \mathrm{ml})$ for $3 \mathrm{~h}$ with frequent agitation. The extract was filtered through absorbent cotton wool. The filter plug and associated solids were further extracted overnight in acetone $(200 \mathrm{ml})$. Combined acetone extracts after filtration were taken to dryness under reduced pressure, and represented at least $95 \%$ of the amount of products of interest which were extractable by an exhaustive protocol. The residue from $P$. crustosum extracts was treated with methanol $(3 \times 15 \mathrm{ml})$ and the extract made up to $100 \mathrm{ml}$. $P$. paxilli extract was processed further by preparative layer silica chromatography.

(iii) Extraction of lysergic acid $\alpha$-hydroxyethylamide. C. paspali culture filtrate $(100 \mathrm{ml})$ was adjusted to $\mathrm{pH} 8.5$ with $\mathrm{NH}_{4} \mathrm{OH}$ and extracted with chloroform $(2 \times 100 \mathrm{ml})$. The organic solvent extract was taken to dryness in vacuo and stored in the dark at $4{ }^{\circ} \mathrm{C}$.

(iv) Extraction of paspalinines. The relatively non-polar indole-diterpenoid paspalinines were retained principally within the mycelium of $C$. paspali. Exploratory extraction of freeze-dried mycelium $(\sim 1 \mathrm{~g})$ using butyl acetate (Gallagher et al., 1980a) proved less efficient than chloroform/acetone $(1: 1, \mathrm{v} / \mathrm{v})$ or acetone $(2 \times 100 \mathrm{ml})$. The latter was preferred since it avoided the possibility of paspalinine degradation by any acid present in chloroform.

The first acetone extraction was over $3 \mathrm{~h}$; the second was overnight. Combined extracts were taken to dryness in vacuo, the residue treated with methanol $(3 \times 10 \mathrm{ml})$ and made up to $50 \mathrm{ml}$.

(v) Isolation of Penicillium metabolites. Efficient separation of penitrem A and roquefortine, prior to HPLC, was achieved using silica Sep-pak cartridges (Waters Associates). The standard methanolic extract was taken to dryness and the solids treated with diethyl ether $(1 \mathrm{ml})$. The extract was loaded on to the cartridge which was eluted 
first with chloroform $(2 \times 1 \mathrm{ml})$ and then acetone $(5 \times 1 \mathrm{ml})$. Eluate fractions were taken to dryness, redissolved in methanol $(100 \mu \mathrm{l})$ and their composition explored by HPLC. The first two drops of eluate contained non-polar substances. The next $2 \mathrm{ml}$ contained penitrems $\mathrm{A}$ and $\mathrm{E}$, completely eluted since the following $1 \mathrm{ml}$ contained neither penitrems nor roquefortine. The next $3 \mathrm{ml}$ contained the roquefortine and iso-roquefortine.

Paxilline was isolated from the acetone extract by preparative chromatography using silica plates and elution of the paxilline band $\left(R_{F} \sim 0.5\right)$ prior to purification by HPLC.

(vi) Isolation of paspalinines. C. paspali mycelial extract in methanol was taken to dryness, the residue was dissolved in chloroform $(500 \mu \mathrm{l})$ and loaded on a $20 \times 20 \mathrm{~cm}$ preparative layer silica plate. The chromatogram was developed in chloroform/acetone $(93: 7, \mathrm{v} / \mathrm{v})$. Prominent bands, evident during irradiation at $254 \mathrm{~nm}$ and confirmed by spraying the edge of the chromatogram with $50 \%$ ethanolic $\mathrm{H}_{2} \mathrm{SO}_{4}$, with $R_{F}$ values 0.61 and 0.23 were paspalinine $\left(M^{+}=433\right)$ and 3-hydroxy-3-methylbutenyl paspalinine $\left(M^{+}=517\right)$, respectively. The latter was the more abundant and was therefore the main focus of attention for biosynthetic studies. Silica of the appropriate bands was eluted with methanol to remove the paspalinines which were purified by HPLC using the Ultrasphere ODS column, methanol/water $(4: 1, \mathrm{v} / \mathrm{v})$ at a flow rate of $4 \mathrm{ml} \mathrm{min}^{-1}$, injection volume $20-50 \mu \mathrm{l}$ and UV detection at $227 \mathrm{~nm}$. 3-Hydroxy-3-methylbutenyl paspalinine eluted 3.5-4.0 min after injection.

(vii) Assay for total penitrems. Standard methanolic extract, or a suitable dilution $(3 \mathrm{ml})$ was mixed with concentrated $\mathrm{H}_{2} \mathrm{SO}_{4}(0.18 \mathrm{ml})$ in a sealed glass tube and incubated at $70^{\circ} \mathrm{C}$ for $20 \mathrm{~min}$. The solution was cooled and the blue colour measured at $630 \mathrm{~nm}$ using a Cary 210 spectrophotometer. Total penitrems, expressed as penitrem A, were calculated by reference to a standard curve for penitrem A, linear over the range 0-30 $\mu \mathrm{g} \mathrm{ml}^{-1}$.

(viii) Assay for roquefortine. An HPLC assay used an Ultrasphere ODS column $(250 \times 4.6 \mathrm{~mm})$, methanol/ water $(4: 1, \mathrm{v} / \mathrm{v})$ mobile phase at $1 \mathrm{ml} \mathrm{min}^{-1}$ and UV detection $(325 \mathrm{~nm})$. Standard methanolic extract (10 $\mu 1$ vols) were injected and roquefortine (retention time $8 \mathrm{~min}$ ) estimated from the peak area on a chart recorder $\left(5 \mathrm{~mm} \mathrm{~min}^{-1}\right)$ and by reference to a linear standard curve over the range $0-80 \mu \mathrm{g} \mathrm{ml}^{-1}$.

(ix) Assay for cyclopenin. Prior to measurement of radioactivity, purified cyclopenin in methanolic solution was quantified spectrophotometrically at $290 \mathrm{~nm}\left(\varepsilon=2060 \mathrm{M}^{-1} \mathrm{~cm}^{-1}\right.$; Martin et al., 1969).

(x) Assay for lysergic acid derivatives in fermentation broth. The principal lysergic acid derivative produced by C. paspali in submerged fermentation is the $\alpha$-hydroxyethylamide. Expressed as the latter, by reference to a linear standard curve over the range $0-100 \mu \mathrm{g} \mathrm{ml}^{-1}$, the total lysergic acid derivatives were determined by mixing culture filtrate $(5 \mathrm{ml})$ with Van Urk reagent $\left[0 \cdot 125 \%\right.$-dimethylaminobenzaldehyde in $65 \%(\mathrm{v} / \mathrm{v}) \mathrm{H}_{2} \mathrm{SO}_{4}$ and $0 \cdot 1 \mathrm{ml} 5 \%$ (v/v) $\mathrm{FeCl}_{3} ; 10 \mathrm{ml}$ and measuring the absorbance after $10 \mathrm{~min}$ at $570 \mathrm{~nm}$ in a Cary 210 spectrophotometer.

(xi) Assay for lysergic acid $\alpha$-hydroxyethylamide. Culture filtrate extract was resolved and lysergic acid $\alpha$-hydroxyethylamide quantified by HPLC using a Waters Radialpak $\mu$-bondapak $\mathrm{C}_{18}$ cartridge in a Z-module, acetonitrile/0.01 $\mathrm{M}-\left(\mathrm{NH}_{4}\right)_{2} \mathrm{CO}_{3}(4: 1 ; \mathrm{v} / \mathrm{v})$ mobile phase at a flow rate of $3 \mathrm{ml} \mathrm{min}{ }^{-1}$. Injection volume was $20 \mu \mathrm{l}$ (methanol). The retention time of the alkaloid was approximately $10 \mathrm{~min}$. Detection by UV absorption $(312 \mathrm{~nm})$ or fluorescence (excitation wavelength $340 \mathrm{~nm}$, emission wavelength $450 \mathrm{~nm}$ ) was linear over the range $0-150 \mu \mathrm{g} \mathrm{ml}^{-1}$.

(xii) Assay for paspalinines. It was necessary to develop analytical methods for paspalinines since none is given in the literature. Paspalinines, like penitrems, give a blue colour when methanolic solutions are heated with $\mathrm{H}_{2} \mathrm{SO}_{4}$, the absorbance maxima being 235 and $656 \mathrm{~nm}$. The same solution fluoresces with excitation and emission maxima at 338 and $415 \mathrm{~nm}$, respectively (Farrand scanning fluorimeter). For a $3 \mathrm{ml}$ methanolic solution of paspalinine the optimum amount of $\mathrm{H}_{2} \mathrm{SO}_{4}$ was $250 \mu \mathrm{l}$ or $100 \mu \mathrm{l}$ for $\mathrm{UV}$ spectrophotometry or fluorimetry, respectively. Mixtures were incubated at $70^{\circ} \mathrm{C}$ for $40 \mathrm{~min}$.

The spectrophotometric assay was marginally more sensitive, giving a linear response over the range $0-15 \mu \mathrm{g}$ paspalinine $\mathrm{ml}^{-1}$; the upper value gave an absorbance of 0.36 at $656 \mathrm{~nm}$. The concentration of paspalinine and its derivatives in mycelial extracts measured using this method was expressed as paspalinine.

(xiii) Assay for 3-hydroxy-3-methylbutenyl paspalinine. Although the maximum UV absorbance wavelengths for this paspalinine derivative have been given (Cole et al., 1977) no molar absorption coefficients were quoted. Thus we measured these values in a standard methanolic solution of purified alkaloid; $\lambda \max .227 \mathrm{~nm}$ $\left(\varepsilon=23100 \mathrm{M}^{-1} \mathrm{~cm}^{-1}\right), 248 \mathrm{~nm}\left(\varepsilon=28500 \mathrm{M}^{-1} \mathrm{~cm}^{-1}\right), \quad 305 \mathrm{~nm}\left(\varepsilon=7100 \mathrm{M}^{-1} \mathrm{~cm}^{-1}\right)$. The molar absorption coefficient at $248 \mathrm{~nm}$ was used to measure the concentration in samples purified for radioactivity measurement.

(xiv) Paxilline. Paxilline was purified by HPLC using a Waters amino $\mu$-bondapak cartridge, dichloromethane/ 2-propanol $(100: 1, \mathrm{v} / \mathrm{v})$ mobile phase at a flow rate of $4 \mathrm{ml} \mathrm{min}^{-1}$, and $\mathrm{UV}$ detection at $281 \mathrm{~nm}$. Injection of $200 \mu \mathrm{l}$ extract in chloroform separated paxilline with a retention time of $3.5 \mathrm{~min}$. Quantitation used $20 \mu \mathrm{l}$ extract and reference to a linear standard curve over the range $0-150 \mu \mathrm{g} \mathrm{ml}^{-1}$.

\section{RESULTS AND DISCUSSION}

\section{Penicillium crustosum fermentation}

The time course of the fermentation of $P$. crustosum in stationary liquid culture when grown at $27{ }^{\circ} \mathrm{C}$ on Czapek Dox broth supplemented with either $0.5 \%$ or $2 \%$ yeast extract showed similar 


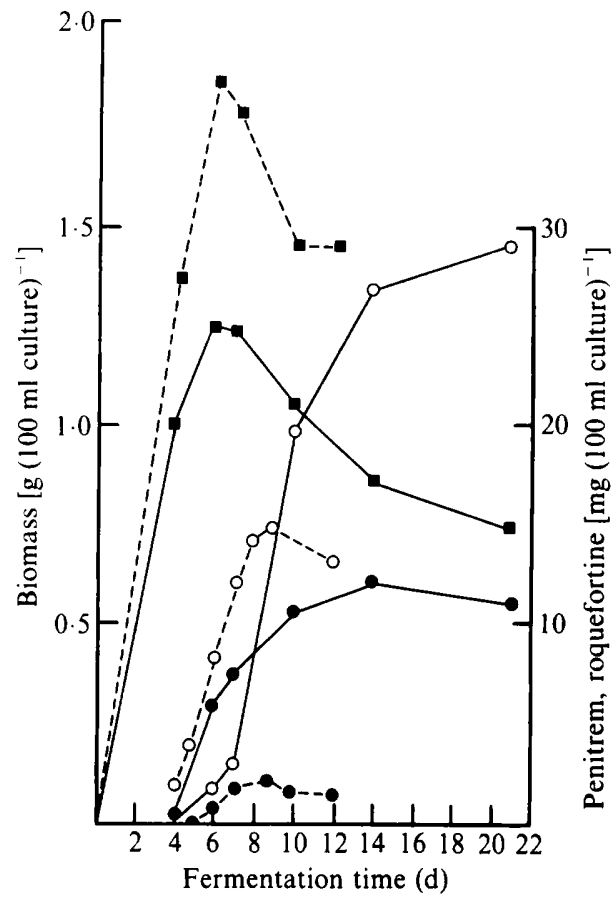

Fig. 1. Progress of stationary liquid culture fermentation of $P$. crustosum producing indole alkaloids on CDYE $(0.5 \%)$ broth (solid lines; $\boldsymbol{\square}$, biomass; $\bigcirc$, roquefortine; $\bullet$, penitrem) and on CDYE $(2 \%)$ broth (dashed lines; $\boldsymbol{\square}$, biomass; $O$, roquefortine; $\bullet$, penitrem). Values are means of triplicate cultures.

dynamics, maximum biomass occurring at $6 \mathrm{~d}$ (Fig. 1). As expected the larger amount of yeast extract supported greater biomass, but also gave inferior yields of penitrem and roquefortine. Sporulation was evident on the upper surface of the mycelial mats after $3 \mathrm{~d}$. Yeast extract $(0.5 \%)$ was used in most subsequent experiments in which $\sim 7 \mathrm{mg}$ penitrems and $10 \mathrm{mg}$ roquefortine were produced in each flask culture.

\section{Incorporation of radiolabelled amino acids and mevalonate into penitrems and roquefortine}

In order to obtain good incorporation of ${ }^{14} \mathrm{C}$-labelled putative precursors into secondary metabolites of $P$. crustosum it was assumed that the optimum time to feed the cultures would be as dry weight became maximum, the rate of protein synthesis having declined and secondary metabolite production commenced. Thus, ${ }^{14} \mathrm{C}$-labelled compounds $(5 \mu \mathrm{Ci}$ for each of two flasks) were fed to 5-d stationary liquid cultures of $P$. crustosum which were incubated for a further $2 \mathrm{~d}$. The numerical results of several feeding experiments are shown in Table 1 , but the autoradiograph comparing incorporations of $\left[\right.$ benzene ring $\left.-{ }^{14} \mathrm{C}\right]$ tryptophan and $\left[2-{ }^{14} \mathrm{C}\right] \mathrm{mevalon}$ ate (Fig. 2) illustrates the dilemma which is the basis of the present study. It fails to provide compelling evidence that penitrem indoles are derived directly from tryptophan. In Table 1 the theoretical molar ratio of specific activity is derived from the number of mols of each precursor required to form $1 \mathrm{~mol}$ of product. Validity as a comparative measure relies on biosyntheses being concurrent at constant rates, drawing on a common precursor pool. Such situations are probably rarely achieved completely but in the $P$. crustosum fermentation at least the relevant secondary biosyntheses are quite closely concurrent (Fig. 1). Irrespective of the position of the ${ }^{14} \mathrm{C}$ label in tryptophan this precursor was incorporated into roquefortine with $>20 \%$ efficiency, a high value for a metabolic intermediate with competitive involvement in protein synthesis. The $23.4 \%$ value is also substantially greater than the $10.8 \%$ reported by Steyn \& Vleggaar (1983) for [benzene ring ${ }^{-14} \mathrm{C}$ ]tryptophan incorporated into roquefortine. Similarly, 


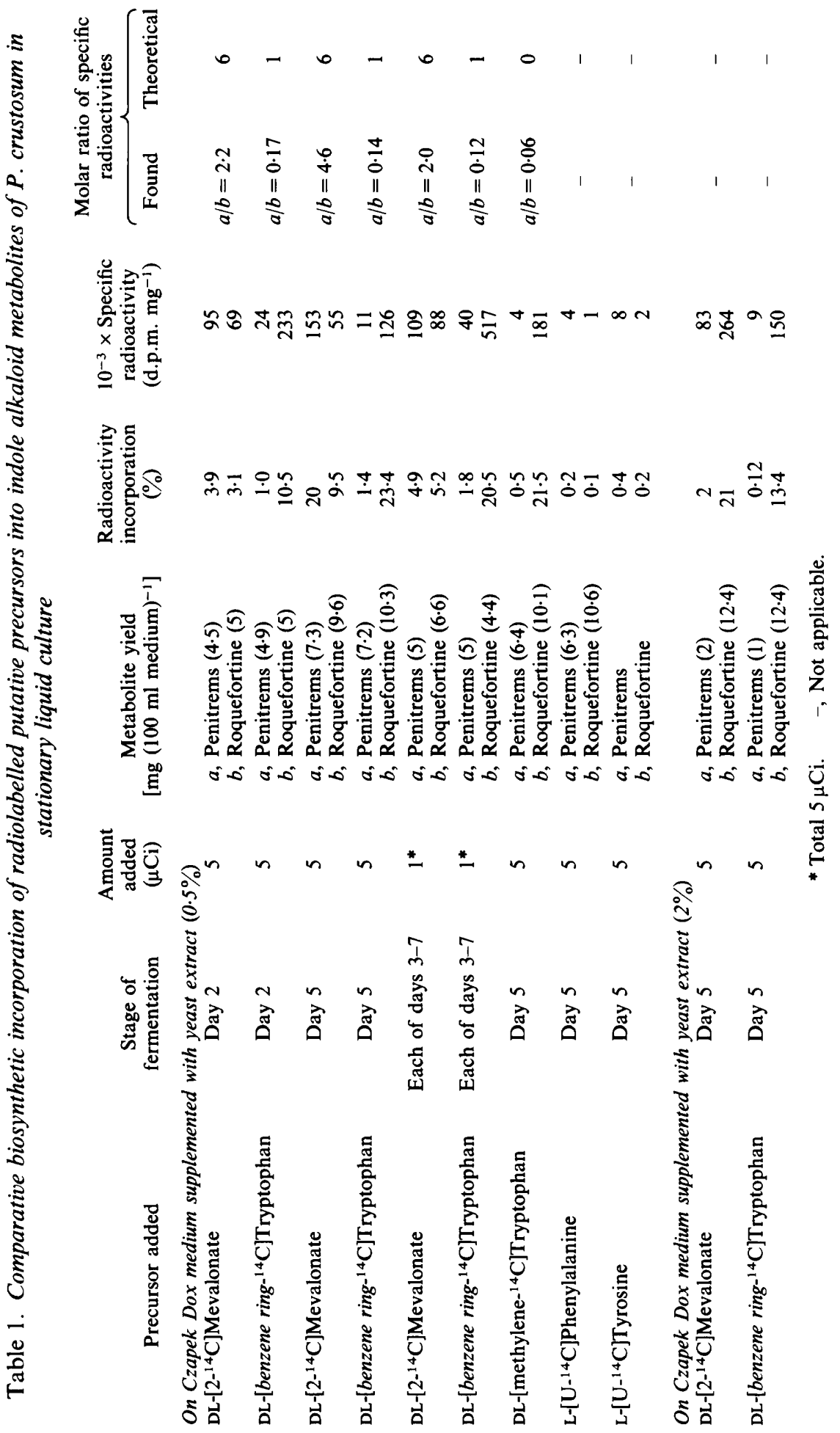




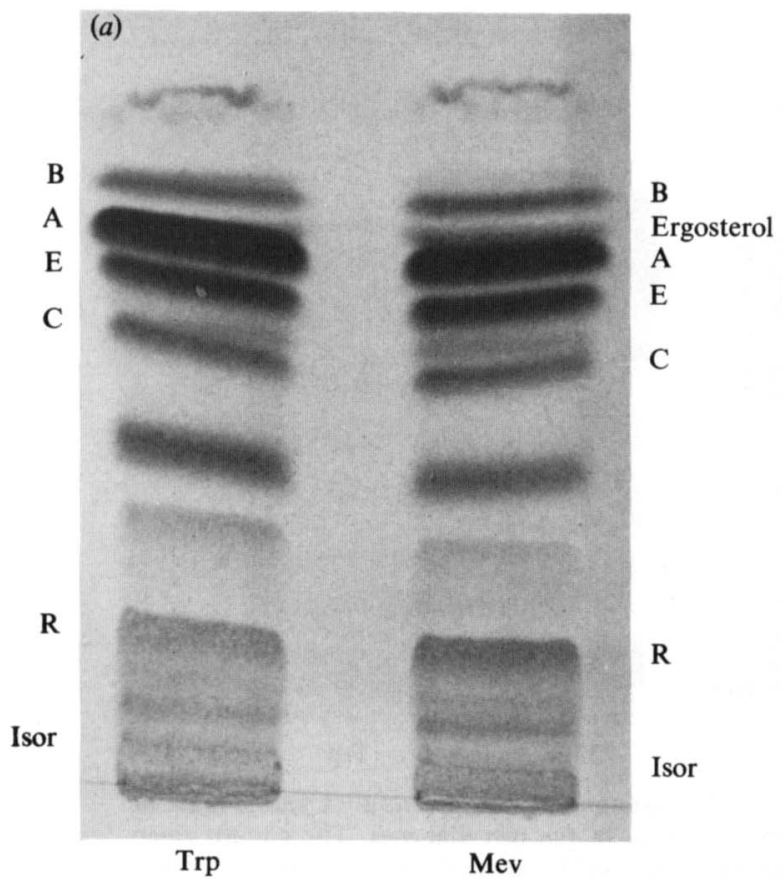

(b)

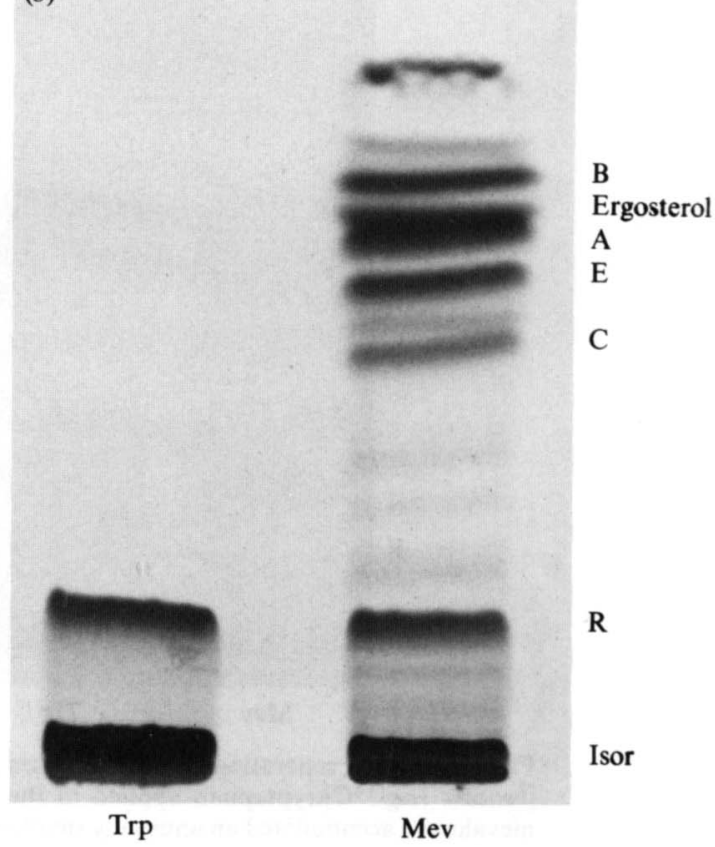

Fig. 2. (a) TLC separation of acetone extracts of $P$. crustosum mycelia given $\left[2-{ }^{14} \mathrm{C}\right] \mathrm{mevalonate}$ or [benzene ring ${ }^{14} \mathrm{C}$ ]tryptophan injected into the fermentation medium. $\mathrm{FeCl}_{3}$ in $\mathrm{n}$-butanol spray reveals, notably, penitrems and roquefortine. (b) Autoradiograph of $(a)$ showing, particularly, disparate radiolabelling of penitrems by $\left[\right.$ benzene ring $\left.-{ }^{14} \mathrm{C}\right]$ tryptophan. Mev, $\left[2-{ }^{14} \mathrm{C}\right]$ mevalonate; Trp, [benzene ring $\left.{ }^{-14} \mathrm{C}\right]$ tryptophan; $\mathrm{A}-\mathrm{E}$, penitrems; $\mathrm{R}$, roquefortine; Isor, isoroquefortine.

prenylation of roquefortine utilized nearly $10 \%$ of the radiolabelled mevalonate. In contrast, although twice as much mevalonate went towards penitrem biosynthesis very little labelled tryptophan was incorporated into penitrems. Only three times more radioactivity in penitrem A was derived from aromatic-labelled tryptophan, as compared with side-chain labelled tryptophan. This hardly constituted grounds for believing that the ${ }^{14} \mathrm{C}$-labelling was by direct incorporation since all the ${ }^{14} \mathrm{C}$ from side-chain-labelled tryptophan has to be acquired by metabolic scrambling. Cleavage of the side-chain of tryptophan by tryptophanase is a required early step in penitrem biosynthesis. The dilemma was further compounded when incorporation of ${ }^{14} \mathrm{C}$ from phenylalanine and tyrosine into penitrem $\mathrm{A}$ was found to be similar in extent to that of side-chain-labelled tryptophan. When product yield and molarities are taken into account the experimental evidence for mevalonate as a precursor of penitrem $\mathrm{A}$ and roquefortine closely approaches $(77 \%)$ the theoretical value whereas [benzene ring $-{ }^{14} \mathrm{C}$ ]tryptophan gave only $14 \%$ of the theoretical value.

Incorporation values of $\left[2-{ }^{14} \mathrm{C}\right]$ mevalonate and [benzene ring $\left.-{ }^{-14} \mathrm{C}\right]$ tryptophan on a medium containing $2 \%$ yeast extract (Table 1 ) were less informative as the penitrem yield was so disproportionately low; the molar ratios of specific radioactivities were $8 \%$ and $10 \%$, respectively, of the theoretical values. However, the percentage incorporation of [benzene ring${ }^{14}$ C]tryptophan $(0 \cdot 12 \%)$ is close to the $0 \cdot 16 \%$ reported by de Jesus et al. (1981). Although not quoted, the yield of penitrem obtained by those authors may be deduced, by extrapolation from de Jesus et al. (1983), to have been 2-4 $\mathrm{mg}$ from the mycelium produced on $100 \mathrm{ml}$ medium. This is of the same order as in the present study.

Cultures fed on day 2, or daily on days 3-6, did not give improved evidence for the origin of the penitrem indole (Table 1). In all circumstances roquefortine, radiolabelled with [benzene ring $\left.-{ }^{14} \mathrm{C}\right]$ tryptophan, had a specific activity an order of magnitude greater than that of penitrem 

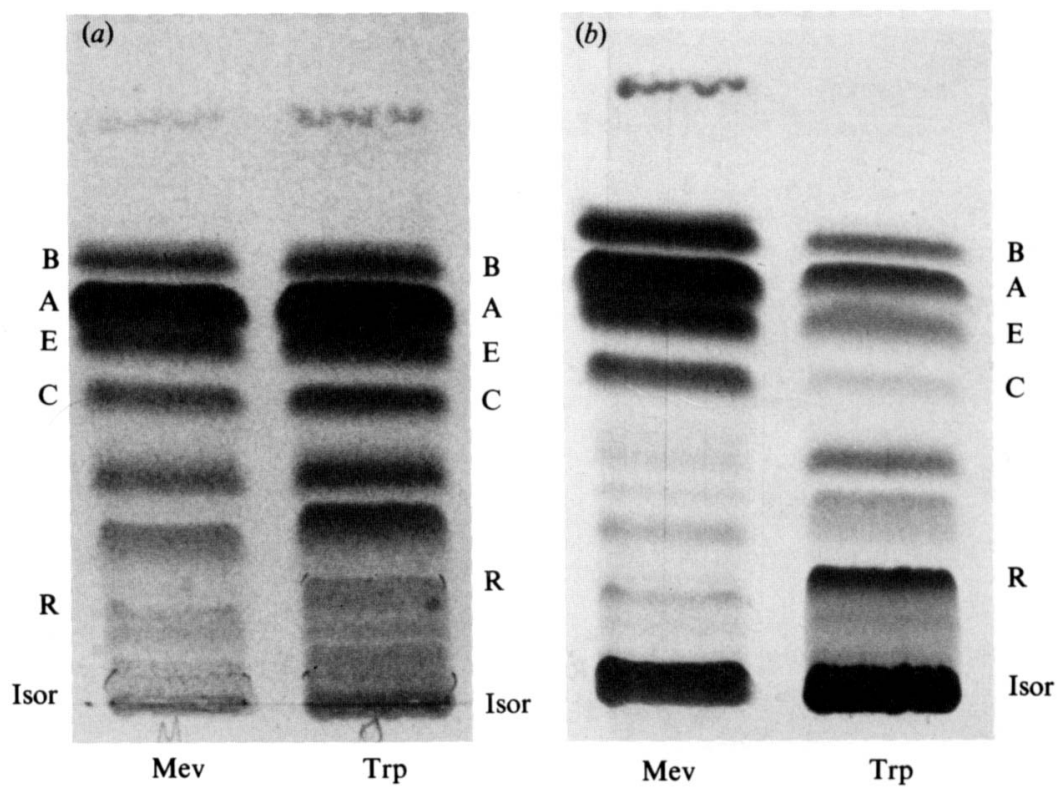

Fig. 3. (a) TLC separation of acetone extracts of $P$. crustosum mycelia given $\left[2{ }^{14} \mathrm{C}\right] \mathrm{mevalonate}$ or [benzene ring ${ }^{-14} \mathrm{C}$ ]tryptophan applied to the upper surface of the mycelial mat. The culture given mevalonate accumulated an unusually small amount of roquefortine, revealed with penitrems by $\mathrm{FeCl}_{3}$ in $\mathrm{n}$-butanol spray. (b) Autoradiograph of $(a)$ showing, in comparison with Fig. 2, improved evidence of the incorporation of [benzene ring $\left.{ }^{-14} \mathrm{C}\right]$ tryptophan into penitrems. Abbreviations, as in Fig. 2.

A, suggesting that tryptophan supplied exogenously to $P$. crustosum is preferentially incorporated into roquefortine because its site(s) of biosynthesis is more accessible.

Polarized differentiation of conidiophores and abstriction of conidia from hyphae furthest away from the nutrient medium during the latter part of the trophophase will accentuate the metabolic gradient across the mycelial mat. Since penitrem biosynthesis is prominent in the spores (Day, 1981), particular secondary metabolite biosynthesis associated with differentiating cells will further polarize the metabolic dynamics across the fungal tissue. Roquefortine biosynthesis is not associated with conidiophore differentiation as is shown by production of the diketopiperazine in non-sporulating submerged cultures (Laws, 1985). Thus radiolabelled putative precursors common to penitrem and roquefortine biosynthesis may be subject to preferential utilization in roquefortine biosynthesis if administered via the medium. However, mevalonate was apparently much less constrained than tryptophan by such polarization with respect to reaching its target. This implied either more active transport of the smaller molecule or incorporation near the site of uptake into, for example, geranylgeranyl pyrophosphate which is then translocated to become involved in penitrem biosynthesis in a more distal part of the fungal mycelium.

\section{Incorporation of tryptophan precursors into the penitrems, roquefortine and cyclopenin}

The results of the preceding experiments left open the possibility that the penitrem indole is not directly derived from tryptophan. Small amounts of ${ }^{14} \mathrm{C}$ from the side-chain of tryptophan, phenylalanine and tyrosine were incorporated into penitrems presumably through metabolic recycling through pathways leading to acetate, phosphoenolpyruvate and/or erythrose 4-phosphate. The extent of such metabolic scrambling was tested using [carboxyl $\left.-{ }^{14} \mathrm{C}\right]-$ anthranilic acid from which the radiolabel is lost during the formation of tryptophan but is retained where the whole anthranilic carbon skeleton becomes a part of a benzodiazepine secondary metabolite. Conveniently, the strain of $P$. crustosum used also elaborates the benzodiazepine cyclopenin into which the radiolabelled anthranilate was incorporated $(0.3 \%)$ 
Table 2. Comparative biosynthetic incorporation of radiolabelled precursors of tryptophan into alkaloid metabolites of P. crustosum in stationary liquid culture

\begin{tabular}{|c|c|c|c|c|c|c|}
\hline \multirow{2}{*}{$\begin{array}{l}\text { Precursor } \\
\text { added }\end{array}$} & \multirow{2}{*}{$\begin{array}{l}\text { Amount } \\
\text { added }^{*} \\
(\mu \mathrm{Ci})\end{array}$} & \multirow{2}{*}{$\begin{array}{l}\text { Metabolite yield } \\
{[\mathrm{mg}(100 \mathrm{ml}} \\
\left.\text { medium })^{-1}\right]\end{array}$} & \multirow{2}{*}{$\begin{array}{l}\text { Radioactivity } \\
\text { incorporation } \\
(\%)\end{array}$} & \multirow{2}{*}{$\begin{array}{c}10^{-3} \times \text { Specific } \\
\text { radioactivity } \\
\left(\text { d.p.m. } \mathrm{mg}^{-1}\right)\end{array}$} & \multicolumn{2}{|c|}{$\begin{array}{c}\text { Molar ratio of specific } \\
\text { radioactivities }\end{array}$} \\
\hline & & & & & Found & Theoretica \\
\hline $\begin{array}{l}\left.\mathrm{D}-[2,3,4,5-(\mathrm{n}))^{-14} \mathrm{C}\right]- \\
\text { Shikimic acid }\end{array}$ & 10 & $\begin{array}{l}a, \text { Penitrems }(9 \cdot 7) \\
b, \text { Roquefortine }(10 \cdot 3) \\
c, \text { Cyclopenin }(3)\end{array}$ & $\begin{array}{l}0 \cdot 3 \\
0 \cdot 95 \\
0 \cdot 24\end{array}$ & $\begin{array}{r}3 \\
10 \\
20\end{array}$ & $\begin{array}{l}a / b=0.53 \\
a / c=0.36 \\
b / c=0.67\end{array}$ & $\begin{array}{l}1 \\
0 \cdot 5 \\
0 \cdot 5\end{array}$ \\
\hline $\begin{array}{l}\left.\text { [carboxyl- }{ }^{14} \mathrm{C}\right]- \\
\text { Anthranilic acid }\end{array}$ & 50 & $\begin{array}{l}a, \text { Penitrems (4) } \\
b \text {, Roquefortine (3) } \\
c \text {, Cyclopenin (3) }\end{array}$ & $\begin{array}{l}0.016 \\
0.02 \\
0.3\end{array}$ & $\begin{array}{r}1 \\
3 \\
37\end{array}$ & $\begin{array}{l}- \\
- \\
-\end{array}$ & $\begin{array}{l}- \\
- \\
-\end{array}$ \\
\hline
\end{tabular}

* Combined amount added to two flasks on day 5, subsequently worked up together on day 7 .

- , Not applicable.

Table 3. Comparative biosynthetic incorporation of radiolabelled precursors into indole alkaloid metabolites of $P$. crustosum in stationary liquid culture, the precursors applied to the fungal mat

\begin{tabular}{|c|c|c|c|c|c|c|}
\hline \multirow{2}{*}{$\begin{array}{l}\text { Precursor } \\
\text { added }\end{array}$} & \multirow{2}{*}{$\begin{array}{c}\text { Amount } \\
\text { added } \\
(\mu \mathrm{Ci})\end{array}$} & \multirow{2}{*}{$\begin{array}{l}\text { Metabolite yield } \\
{[\mathrm{mg}(100 \mathrm{ml}} \\
\left.\text { medium })^{-1}\right]\end{array}$} & \multirow{2}{*}{$\begin{array}{c}\text { Radioactivity } \\
\text { incorporation } \\
(\%)\end{array}$} & \multirow{2}{*}{$\begin{array}{c}10^{-3} \times \text { Specific } \\
\text { radioactivity } \\
\left(\text { d.p.m. } \mathrm{mg}^{-1}\right)\end{array}$} & \multicolumn{2}{|c|}{$\begin{array}{l}\text { Molar ratio of specific } \\
\text { radioactivities }\end{array}$} \\
\hline & & & & & Found & Theoretical \\
\hline $\begin{array}{l}\text { DL- }\left[2-^{-14} \mathrm{C}\right]- \\
\text { Mevalonate }\end{array}$ & 10 & $\begin{array}{l}a \text {, Penitrems }(8.7) \\
b, \text { Roquefortine }(3.4)\end{array}$ & $\begin{array}{l}15 \cdot 5 \\
11 \cdot 7\end{array}$ & $\begin{array}{l}197 \\
400\end{array}$ & $a / b=0.8$ & 6 \\
\hline $\begin{array}{l}\text { DL- }\left[\text { benzene ring }-{ }^{-14} \mathrm{C}\right]- \\
\text { Tryptophan }\end{array}$ & 10 & $\begin{array}{l}a, \text { Penitrems (10) } \\
b \text {, Roquefortine (4) }\end{array}$ & $\begin{array}{l}3 \cdot 6 \\
10\end{array}$ & $\begin{array}{r}40 \\
278\end{array}$ & $a / b=0.23$ & 1 \\
\hline
\end{tabular}

(Table 2). The modest incorporation value reflects the demands of active tryptophan biosynthesis even after maximum biomass had been formed. This is consistent with a need to elaborate tryptophan for post-trophophase penitrem biosynthesis, if penitrem is derived from tryptophan. Consequently, part of the ${ }^{14} \mathrm{CO}_{2}$ released re-enters metabolism through anaplerotic pathways and both penitrem $A$ and roquefortine acquire radiolabel equivalent to about $0.02 \%$ incorporation of $\left[\right.$ carboxyl $\left.{ }^{-14} \mathrm{C}\right]$ anthranilate. This value is close to that reported by de Jesus et al . (1981) for incorporation of [methylene $\left.{ }^{-14} \mathrm{C}\right]$ tryptophan into penitrem A. Thus, the credibility gap in experimentation was still whether an eightfold increase above a spurious value is a basis for confidence.

Incorporations of $\left[2,3,4,5-{ }^{14} \mathrm{C}\right]$ shikimate into penitrem $\mathrm{A}$, roquefortine and cyclopenin (Table 2 ), interpreted in conjunction with the molar ratios of their specific radioactivities, were reasonably in accord with the theoretical values considering experimental limitations. It was concluded that the results are consistent with these secondary metabolites containing moieties derived directly from the common part of the aromatic amino acid biosynthetic pathway.

\section{Administration of radiolabelled precursors via the upper surface of mycelia}

Radiolabelled precursors were administered on day 5 on the layer of hydrophobic spores which form the upper surface of $P$. crustosum mycelia. Precursors were applied in $200 \mu 10.01 \%$ Tween 80 as ten spatially separate drops of $20 \mu \mathrm{l}$ each. Cultures were harvested $2 \mathrm{~d}$ later. The results (Table 3, Fig. 3) showed greatly improved numerical and autoradiographic evidence consistent with the role of tryptophan as the direct precursor of the penitrem indole. This was in spite of the obvious disadvantage of impaired uptake of precursors from droplets by the unwettable $P$. crustosum spores, the most mature of which would have been those immediately adjacent to the applied drops. Whereas this form of polarized application of precursor allowed relative mevalonate incorporation to reach only $13 \%$ of the 'theoretical' value of the molar ratio of specific radioactivities of penitrems and roquefortine, incorporation of [benzene ring${ }^{14}$ C]tryptophan reached $23 \%$ of the 'theoretical' value. 


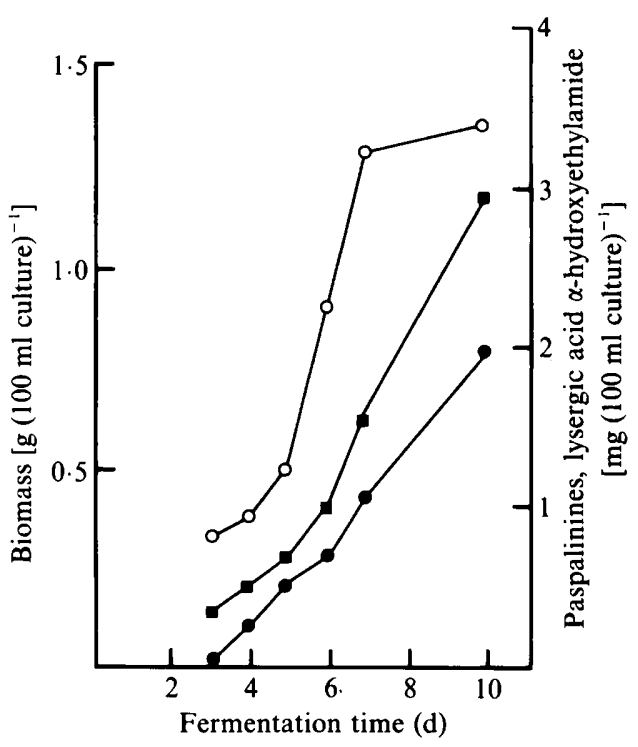

Fig. 4. Progress of submerged culture fermentation of $C$. paspali producing indole alkaloids. $\square$, Biomass; O, lysergic acid $\alpha$-hydroxyethylamide; $\boldsymbol{O}$, paspalinines. Values are means of triplicate cultures.

\section{Claviceps paspali fermentation}

C. paspali offers a fermentation in which a substituted indole-diterpenoid analogous to penitrem $\mathrm{A}$ is found but in which another metabolite, derived directly from tryptophan, also occurs. Biosynthesis of the diterpenoid part of the indole-diterpenoid paspaline and derivatives has been studied by Acklin et al. (1977), presumably in submerged fermentation although no fermentation details were published. C. paspali is also a well-known source of the ergot alkaloid lysergic acid $\alpha$-hydroxyethylamide in submerged fermentation, a classic example of a metabolite derived from the whole carbon skeleton of tryptophan except the carboxyl carbon. The suitability of $C$. paspali as an experimental alternative to $P$. crustosum was demonstrated (Fig. 4) in the concurrent production of lysergic acid $\alpha$-hydroxyethylamide and paspalinine indolediterpenoids. A disadvantage for biosynthetic studies was the potentially high competition for added precursors from replicatory growth processes, but an advantage was the more homogeneous nature of the fermentation which avoided the polarized dynamics of stationary liquid culture.

\section{Incorporation of radiolabelled mevalonate and tryptophan into C. paspali alkaloids}

Percentage incorporations of mevalonate into the two metabolites (Table 4) were consistent with their isoprenoid derivation in competition with the demands of sterol biosynthesis during cellular replication. Of the added [benzene ring ${ }^{14} \mathrm{C}$ ]tryptophan, $16 \cdot 3 \%$ was diverted to lysergyl biosynthesis [less than the amount incorporated into roquefortine (Table 1)] but $2.6 \%$ was incorporated into the indole-diterpenoid. Further, the molar ratios of specific radioactivities deviated in a similar range $(50-60 \%)$ from the theoretical values for incorporation of the two precursors studied. Thus, more convincing experimental evidence for the origin of the indole moiety from tryptophan was becoming evident, and coincided with the publication of definitive ${ }^{13} \mathrm{C}$ evidence that the penitrem $\mathrm{A}$ indole is derived directly from tryptophan (de Jesus et al., 1983). It was concluded that the more homogeneous submerged culture system was more amenable to meaningful experimentation and thus an efficient submerged fermentation system for production of the indole-diterpenoid paxilline was explored. 


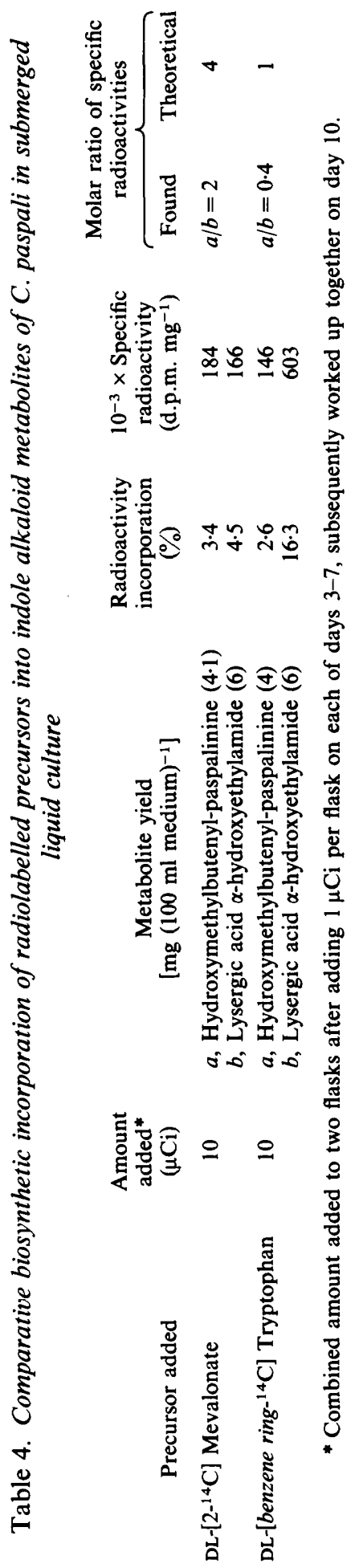




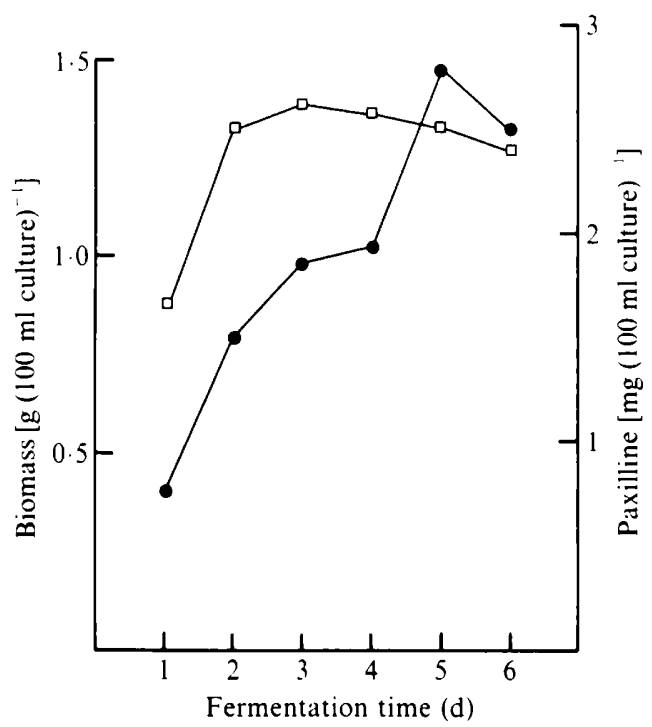

Fig. 5. Progress of submerged culture fermentation of $P$. paxilli producing an indole-diterpenoid alkaloid. $\square$, Biomass; $\bullet$, paxilline. Values are means of triplicate cultures.

Incorporation of [benzene ring $\left.-{ }^{14} \mathrm{C}\right]$ tryptophan into paxilline biosynthesis by Penicillium paxilli

During the course of biomass accumulation and paxilline production in shaken flask cultures (Fig. 5), [benzene ring $-{ }^{14} \mathrm{C}$ ]tryptophan $(2 \mu \mathrm{Ci})$ was fed to two cultures; $17 \%, 53 \%$ and $30 \%$ of the radiolabel was given towards the end of the trophophase at $40 \mathrm{~h}, 50 \mathrm{~h}$ and $60 \mathrm{~h}$, respectively. The cultures were harvested at $5 \mathrm{~d}$. Autoradiography of a TLC of the mycelial extract showed that paxilline was the only component which was prominently radiolabelled. In each flask the mean yield of paxilline was $2.5 \mathrm{mg}$ and radioactivity levels corresponded to incorporation of $5 \%$ of the racemic radiolabelled tryptophan.

\section{Aspects of the dynamics of roquefortine and penitrem biosynthesis}

Duplicate $P$. crustosum surface cultures were fed $\left[2-{ }^{14} \mathrm{C}\right]$ histidine $(4 \mu \mathrm{Ci})$ and $\left[2-{ }^{3} \mathrm{H}_{3}\right]$ mevalonate $(50 \mu \mathrm{Ci})$ on either days $2,3,4,5$ or 6 . All cultures were harvested on day 7 . Concurrently, similar cultures were sampled to follow the course of growth and metabolite accumulation (Fig. $6 a$ ). The specific activities of roquefortine with respect to the two nuclides ${ }^{14} \mathrm{C}$ and ${ }^{3} \mathrm{H}$ labelling the histidinyl and isoprene moieties, respectively, showed divergent dynamics over the early period of roquefortine accumulation (Fig. 6b). The implied temporal separation of initial diketopiperazine formation from subsequent prenylation was more clearly evident in plotting the ratios of the specific activities (Fig. $6 d$ ) and is similar to that demonstrated in the analogous diketopiperazine verruculogen (Mantle \& Shipston, 1987).

The steady increase in specific activity of penitrem A (Fig. $6 c$ ), labelled by ${ }^{3} \mathrm{H}$ in isoprenes, may be partly in response to progressively reduced demand for sterol biosynthesis as biomass reaches a maximum. The significant incorporation, even when the label was given on day 2 and before penitrem is a detectable metabolite, may indicate a preformed pool of geranylgeranyl pyrophosphate destined for later use in the diterpenoid moiety of penitrem $A$. The fivefold increase in specific activity from the day 6 feed, compared with the day 2 feed, is evidence for the later addition of isoprene units to the benzene ring of the indole moiety after the formation of a paxilline-like precursor containing the diterpenoid skeleton. These isoprenes retain five ${ }^{3} \mathbf{H}$ atoms from $\left[2-{ }^{3} \mathrm{H}_{3}\right]$ mevalonate. Plotting the molar ratio of specific activities of penitrem and roquefortine with respect to $\left[{ }^{3} \mathrm{H}\right]$ mevalonate (Fig. $6 d$ ) shows a temporary surge in incorporation into penitrem after days 3 and 4 coinciding with the acceleration in biosynthesis of this indoleisoprenoid. The expected theoretical ratio between the molar specific activities of penitrem $A$ 

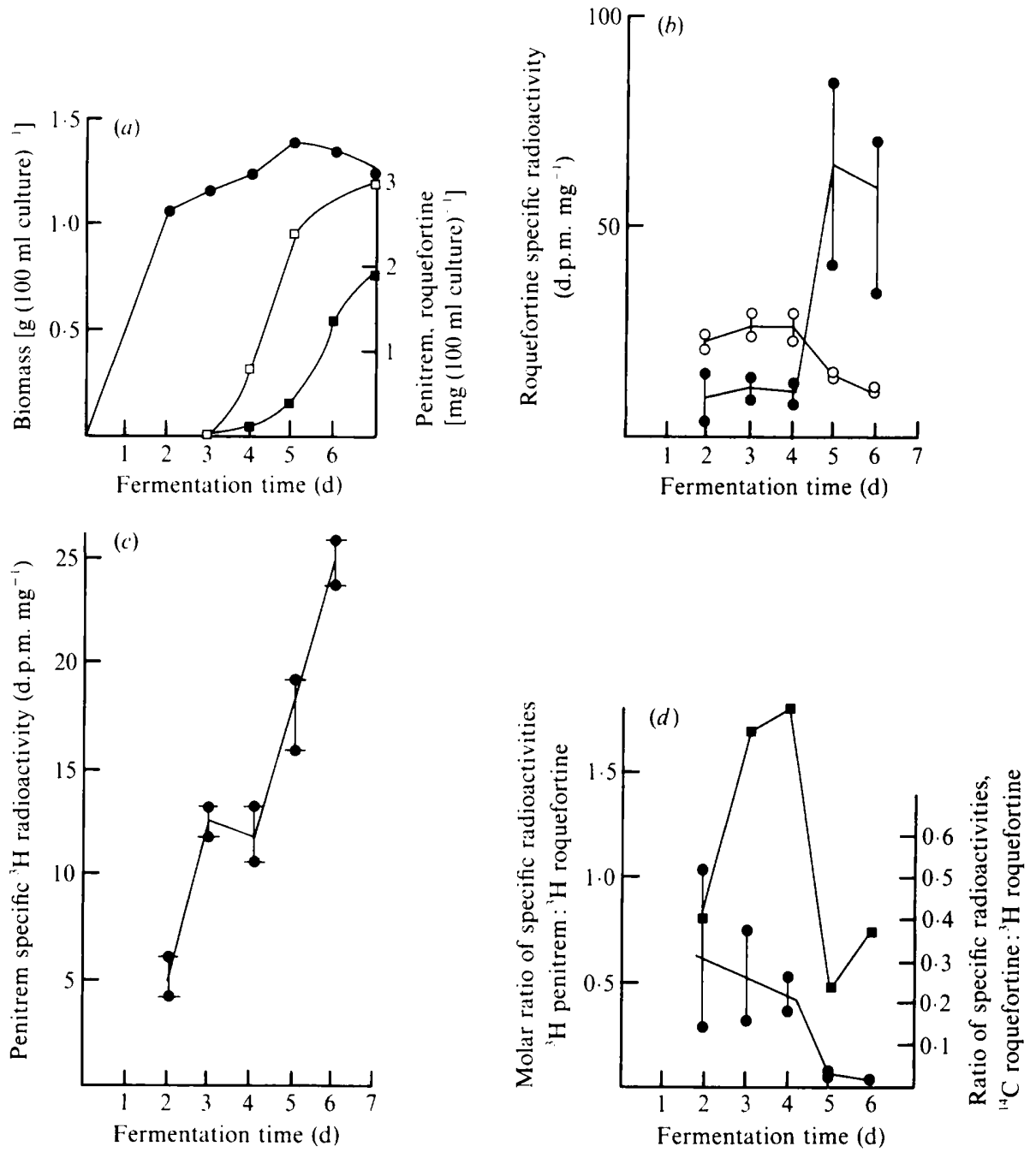

Fig. 6. (a) Progress of indole alkaloid production in stationary liquid culture fermentation of $P$. crustosum during an experiment to measure relative incorporations of biosynthetic precursors. $\bullet$, Biomass; $\square$, penitrem; $\boldsymbol{\square}$, roquefortine. Values are means of duplicate cultures. (b) Change in specific radioactivities of roquefortine with respect to ${ }^{3} \mathrm{H}$ and ${ }^{14} \mathrm{C}$ at day 7 following administration of a standard mixture of $\left[{ }^{14} \mathrm{C}\right]$ histidine and $\left[{ }^{3} \mathrm{H}\right]$ mevalonate on each of days 2-6 during the course of fermentation. $-\left(10^{-5} \times\right)$ specific ${ }^{3} \mathrm{H}$ radioactivity; $\mathrm{O},\left(10^{-4} \times\right)$ specific ${ }^{14} \mathrm{C}$ radioactivity. (c) Change in $\left(10^{-5} \times\right)$ specific ${ }^{3} \mathrm{H}$ radioactivities of penitrem, corresponding to $(b)$ above. $(d)$ Change in the ratios () of the specific radioactivities of roquefortine with respect to ${ }^{14} \mathrm{C}$ and ${ }^{3} \mathrm{H}$, and the molar ratios $(\boldsymbol{\square})$ of the specific radioactivities of penitrem $\mathrm{A}$ and roquefortine with respect to ${ }^{3} \mathbf{H}$, during the course of fermentation.

and roquefortine, with respect to the atoms persisting from $\left[2-{ }^{3} \mathrm{H}_{3}\right]$ mevalonate, is $4: 1$ (de Jesus et al., 1983; Barrow et al., 1979). The disparate rates of biosynthesis of the two types of metabolite up to day 7 (Fig. $6 a$ ), although both biosyntheses are quite closely concurrent, did not allow experimental expression of more than 1.7:1 even at the optimum stage of administration (day 4).

In conclusion, biosynthesis of secondary metabolites in filamentous fungi should, if possible, be studied in submerged fermentation to avoid the polarized endogenous dynamics of surfacecultured biomass and to optimize uptake of exogenously administered precursors. In comparing 
concurrent biosynthesis of different types of molecule drawing on common precursors the possibility of differential sub-cellular compartmentation of the particular enzymes for the particular biosyntheses should be recognized when interpreting the experimental fate of putative precursors.

We wish to thank the Science and Engineering Research Council for a Research Studentship (I.L.).

\section{REFERENCES}

Acklin, W., Weibel, F. \& Arigoni, D. (1977). Zur Biosynthese von Paspalin und verwandten Metaboliten aus Claviceps paspali. Chimia 31, 63.

Barrow, K. D., Colley, P. W. \& Tribe, D. E. (1979). Biosynthesis of the neurotoxin alkaloid roquefortine. Journal of the Chemical Society Chemical Communications, 225-226.

COle, R. J., Dorner, J. W., LANSDEN, J. A., CoX, R. H., Pape, C., Cunfer, B., Nicholson S. S. \& BEDELL, D. M. (1977). Paspalum staggers: isolation and identification of tremorgenic metabolites from sclerotia of C. paspali. Journal of Agricultural and Food Chemistry 25, 1197-1201.

DAY, J. B. (1981). Tremorgenic mycotoxins and natural staggers syndromes. $\mathrm{PhD}$ thesis, University of London.

Gallagher, R. T., Finer, J., Clardy, J., Leutwiler, A., Weibel, F., ACKLIN, W. \& ARIGONI, D. (1980a). Paspalinine, a tremorgenic metabolite of Claviceps paspali Stevens et Hall. Tetrahedron Letters 21, 235238.

Gallagher, R. T., Clardy, J. \& Wilson, B. J. $(1980 b)$. Aflatoxin, a tremorgenic toxin from Aspergillus flavus. Tetrahedron Letters 21, 239-242.

Gallagher, R. T., Hawkes, A. D., Steyn, P. S. \& VlegGaAR, R. (1984). Tremorgenic neurotoxins from perennial ryegrass causing ryegrass staggers of livestock: structure elucidation of lolitrem B. Journal of the Chemical Society Chemical Communications, 614-616.

IbBa, M., TAYloR, S. J. C., Weedon, C. M. \& Mantle, P. G. (1987). Submerged fermentation of Penicillium paxilli biosynthesizing paxilline, a process inhibited by calcium-induced sporulation. Journal of General Microbiology 133, 3109-3119.

De Jesus, A. E., Steyn, P. S., Van Heerden, F. R., VleggaAR, R., Wessels, P. L. \& Hull, W. E. (1981). Structure and biosynthesis of the penitrems A-F, six novel tremorgenic mycotoxins from Penicillium crustosum. Journal of the Chemical Society Chemical Communications, 289-291.

De Jesus, A. E., Gorst-Allman, C. P., Steyn, P. S.,
Van Heerden, F. R., VleggaAr, R., Wessels, P. L. \& HuLL, W. E. (1983). Tremorgenic mycotoxins from Penicillium crustosum: biosynthesis of penitrem A. Journal of the Chemical Society Perkin Transactions I, 1863-1868.

De Jesus, A. E., Steyn, P. S., Van Heerden, F. R. \& VLEGGAAR, R. (1984). Structure elucidation of the janthitrems, novel tremorgenic mycotoxins from Penicillium janthinellum. Journal of the Chemical Society Perkin Transactions I, 697-701.

LAWS, I. (1985). Biosynthesis and metabolism of indolic fungal metabolites. $\mathrm{PhD}$ thesis, University of London.

Mantle, P. G., Mortimer, P. H. \& White, E. P. (1978). Mycotoxic tremorgens of Claviceps paspali and Penicillium cyclopium: a comparative study of effects on sheep and cattle in relation to natural staggers syndromes. Research in Veterinary Science 24, 49-56.

Mantle, P. G., Perera, K. P. W. C., Maishman, N. J. \& MUNDY, G. R. (1983). Biosynthesis of penitrems and roquefortine by Penicillium crustosum. Applied and Environmental Microbiology 45, 1486-1490.

Mantle, P. G. \& Shipston, N. F. (1987). Temporal separation of steps in the biosynthesis of verruculogen. Biochemistry International 14, 1115-1120.

MARTIN, P. K., RAPOPORT, H., SMith, H. W. \& Wong, L. J. (1969). The synthesis of cyclopenin and isocyclopenin. Journal of Organic Chemistry 34, 1359-1363.

Springer, J. P., Clardy, J., Wells, J. M., Cole, R. J. \& KIRKSEY, J. W. (1975). The structure of paxilline, a tremorgenic metabolite of Penicillium paxilli. Tetrahedron Letters 30, 2531-2534.

STEYN, P. S. \& VlegGaAR, R. (1983). Roquefortine, an intermediate in the biosynthesis of oxaline in cultures of Penicillium oxalicum. Journal of the Chemical Society Chemical Communications, 560-561.

Weedon, C. M. \& Mantle, P. G. (1987). Paxilline biosynthesis by Acremonium loliae; a step towards defining the origin of lolitrem neurotoxins. Phytochemistry 26, 969-971. 